\title{
Bacillus anthracis spore interactions with mammalian cells: Relationship between germination state and the outcome of in vitro
}

\author{
Ian M Gut ${ }^{1 \dagger}$, Batcha Tamilselvam ${ }^{1 \dagger}$, Angela M Prouty ${ }^{1,4}$, Bojana Stojkovic ${ }^{1,5}$, Stephanie Czeschin ${ }^{1}$, \\ Wilfred A van der Donk ${ }^{2,3^{*}}$, Steven R Blanke ${ }^{1,3^{*}}$
}

\begin{abstract}
Background: During inhalational anthrax, internalization of Bacillus anthracis spores by host cells within the lung is believed to be a key step for initiating the transition from the localized to disseminated stages of infection. Despite compelling in vivo evidence that spores remain dormant within the bronchioalveolar spaces of the lungs, and germinate only after uptake into host cells, most in vitro studies of infection have been conducted under conditions that promote rapid germination of spores within the culture medium.

Results: Using an in vitro model of infection, we evaluated the influence of the germination state of $B$. anthracis spores, as controlled by defined culture conditions, on the outcome of infection. Spores prepared from B. anthracis Sterne 7702 germinated in a variety of common cell culture media supplemented with fetal bovine serum (FBS) while, in the absence of FBS, germination was strictly dependent on medium composition. RAW264.7 macrophagelike cells internalized spores to the same extent in either germinating or non-germinating media. However, significantly more viable, intracellular B. anthracis were recovered from cells infected under non-germinating conditions compared to germinating conditions. At the same time, RAW264.7 cells demonstrated a significant loss in viability when infected under non-germinating conditions.
\end{abstract}

Conclusions: These results suggest that the outcome of host cell infection is sensitive to the germination state of spores at the time of uptake. Moreover, this study demonstrates the efficacy of studying B. anthracis spore infection of host cells within a defined, non-germinating, in vitro environment.

\section{Background}

Inhalational anthrax commences with the deposition of Bacillus anthracis spores into the bronchioalveolar spaces of the lungs, and culminates with the systemic dissemination of vegetative bacilli within the host [1-3]. Within the lungs, internalization of dormant spores, possibly by several different types of host cells, is believed to be a key step for initiating the transition from the localized to disseminated stages of infection. Alveolar macrophages are reported to transport spores out of the lungs to regional lymph nodes [4-7].

\footnotetext{
* Correspondence: vddonk@illinois.edu; sblanke@life.uiuc.edu

† Contributed equally

'Department of Microbiology, University of Illinois, Urbana, IL, 61801, USA ${ }^{2}$ Department of Chemistry and Howard Hughes Medical Institute, University of Illinois, Urbana, IL, 61801, USA

Full list of author information is available at the end of the article
}

Dendritic cells have also been implicated in the rapid carriage of spores to the draining lymph nodes $[8,9]$. Finally, alveolar epithelial cells have recently been demonstrated to internalize spores both in vitro and in vivo [10-12], and have been proposed to facilitate the transcytosis of $B$. anthracis across the epithelial barrier. Taken together, these findings suggest that $B$. anthracis may escape the lungs by several distinct mechanisms.

To characterize the interaction of $B$. anthracis spores with host cells during the early stages of inhalational anthrax, in vitro models of infection have been widely implemented [8,13-22]. The tractability of in vitro models has facilitated new insights into the molecular and cellular basis of spore binding and uptake, as well as host cell responses. Nonetheless, the use of in vitro models has resulted in a striking lack of consensus as to the responses and fates of both intracellular B. anthracis

\section{Biomed Central}


and infected cells. Although there are multiple reports of germinated spores within host cells [13,15,16,20,23], several studies have indicated that germinated spores ultimately kill macrophages [13,19,20], while others have reported that macrophages readily kill intracellular $B$. anthracis [21,22]. The lack of consensus may be due, in part, to fundamental differences between the infection models used by research groups, which includes variability in bacterial strains, mammalian cells, and experimental conditions employed.

An important issue that is likely to directly influence the outcome of in vitro models of infection is the germination state of spores as they are internalized into host cells. Several in vivo lines of evidence support the idea that spores remain dormant in the alveolar spaces of the lungs prior to uptake. First, dormant spores have been recovered from the lungs of animals several months after initial infection $[7,24]$. Second, all spores collected from the bronchial alveolar fluids of spore-infected Balb/c mice were found to be dormant $[5,23]$. In contrast, a substantial percentage of intracellular spores recovered from alveolar macrophages were germinated [23]. Third, real time in vivo imaging failed to detect germinated spores within lungs, despite the effective delivery of dormant spores to these organs [25-27]. One of these studies [25] reported that vegetative bacteria detected in the lungs during disseminated $B$. anthracis infection arrived at the lungs via the bloodstream, rather than originating from in situ spore growth. Finally, using spores that had been engineered to emit a bioluminescent signal immediately after germination initiation, a recent study reported that germination was commenced in a mouse model of infection only after spore uptake into alveolar macrophages [6]. However, despite considerable evidence that the lung environment is not intrinsically germinating for $B$. anthracis spores, most in vitro infection models have been conducted using culture media containing FBS and/ or specific L-amino acids or nucleotides at concentrations previously demonstrated to promote germination of spores in vitro [20,28-34]. Under such conditions, it is likely that, in these previous studies, host cells were infected with heterogeneous populations of germinated and dormant spores.

The objective of this study was to experimentally address existing gaps in knowledge as to how the germination state of $B$. anthracis spores, as dictated by the presence or absence of serum during in vitro infections, influences the uptake of spores into mammalian cells, as well as the subsequent fate of both intracellular $B$. anthracis and infected cells. Germinating and non-germinating culture conditions were used to compare the interaction of spores prepared from B. anthracis Sterne 7702 with RAW264.7 macrophage-like cells, as well as several other cell lines. These studies revealed that the uptake of B. anthracis into cells was largely unaffected by the germination state of spores. In contrast, the number of viable, intracellular B. anthracis recovered from infected cells, as well as the viability of the infected cells, was dependent on the germination state of spores during uptake. These results support the idea that the germination state of spores is an important consideration when interpreting results from in vitro infections with B. anthracis spores.

\section{Results and Discussion}

The composition of cell culture medium influences the germination and outgrowth of $B$. anthracis spores

Several commonly used mammalian cell culture media, in the presence or absence of fetal bovine serum (FBS), were first evaluated for the capacity to induce germination initiation, which is the earliest set of changes in dormant spores triggered by the presence of germinants. Spore outgrowth, which is the transition of germinated spores into vegetative bacilli [35-37], was also evaluated. These studies revealed that, regardless of the medium tested, dormant spores prepared from B. anthracis Sterne $7702\left(1.0 \times 10^{8}\right.$ spores $\left./ \mathrm{mL}\right)$ underwent germination initiation when incubated at $37^{\circ} \mathrm{C}$ and under $5 \%$ $\mathrm{CO}_{2}$ in the presence of FBS, as indicated by increased sensitivity of the spores to heat treatment [38] and a time-dependent decrease in spore refractility, which indicates rehydration of the spore core following germination initiation [39] (Table 1, Figure 1A, B). When incubated in Dulbecco's modified Eagle's medium (DMEM) plus 10\% FBS, or, Roswell Park Memorial Institute (RPMI) 1640 medium plus 10\% FBS, $86.0 \pm$ $5.2 \%$ and $83.4 \pm 2.6 \%$ of total spores, respectively, converted from heat-resistant to heat sensitive forms within $10 \mathrm{~min}$, while $97.6 \pm 0.2 \%$ and $96.6 \pm 2.2 \%$ of total spores, respectively, converted to heat sensitive forms within $60 \mathrm{~min}$, as determined by dilution plating and direct CFU counting over the course of three independent experiments. These results are consistent with a previous study reporting that approximately $98 \%$ of the B. anthracis Sterne spores germinated within an hour when incubated in DMEM plus 10\% FBS $[13,20]$. Another previous study reported that when incubated in minimal essential medium (MEM) supplemented with $10 \%$ FBS, approximately $37 \%$ of Sterne spores germinated within one hour [40]. Dose response studies revealed that germination initiation was induced in DMEM containing 1\% FBS, but not $0.5 \%$ FBS (Table 2). Spore germination or outgrowth was not dependent on the commercial source of FBS, as similar results were obtained with FBS purchased from 3 different vendors (data not shown). The capacity of spore preparations to germinate were confirmed by incubating dormant spores in the presence of the known germinants, L-alanine and 
Table 1 Germination and outgrowth of B. anthracis spores as a function of cell culture medium in the presence or absence of FBS $^{a}$

\begin{tabular}{|c|c|c|c|c|}
\hline \multirow[b]{2}{*}{ medium $^{b}$} & \multirow[b]{2}{*}{$\mathrm{FBS}^{\mathrm{c}}$} & \multirow[b]{2}{*}{ germination $^{d}$} & \multicolumn{2}{|c|}{ outgrowth $^{e}$} \\
\hline & & & $1 \mathrm{~h}$ & $4 \mathrm{~h}$ \\
\hline \multirow[t]{2}{*}{ DMEM } & - & - & - & - \\
\hline & + & + & + & + \\
\hline \multirow[t]{2}{*}{ RPMI } & - & - & - & - \\
\hline & + & + & + & + \\
\hline \multirow[t]{2}{*}{ MEM $\alpha$} & - & + & + & + \\
\hline & + & + & + & + \\
\hline \multirow[t]{2}{*}{ MEM } & - & - & - & \\
\hline & + & + & + & + \\
\hline \multirow[t]{2}{*}{ AMEM } & - & - & - & - \\
\hline & + & + & + & + \\
\hline \multirow[t]{2}{*}{ EMEM } & - & - & - & - \\
\hline & + & + & + & + \\
\hline \multirow[t]{2}{*}{ BME } & - & - & - & - \\
\hline & + & + & + & + \\
\hline \multirow[t]{2}{*}{ CIM } & - & + & + & + \\
\hline & + & + & + & + \\
\hline \multirow[t]{2}{*}{$F-12$} & - & - & - & - \\
\hline & + & + & + & + \\
\hline \multirow[t]{2}{*}{ M5A } & - & + & + & + \\
\hline & + & + & + & + \\
\hline $\mathrm{BHI}$ & - & + & + & + \\
\hline LB & - & + & + & + \\
\hline $\mathrm{AA}^{f}$ & - & + & - & - \\
\hline
\end{tabular}

${ }^{a}$ Three independent experiments were performed with three different spore preparations, each conducted in triplicate.

${ }^{b}$ Spores prepared from B. anthracis Sterne 7702 were incubated in the indicated medium.

${ }^{c}$ Indicates the presence $(+)$ or absence $(-)$ of $10 \%$ FBS in the indicated medium

${ }^{d}$ Spores were scored positive (+) for germination if the $\mathrm{OD}_{600 \mathrm{~nm}}$ of the suspended spores decreased by more than $10 \%$ after 30 min incubation in the indicated medium.

${ }^{e}$ Using DIC microscopy, spores were scored positive (+) for outgrowth if the spores bodies were visibly larger at $1 \mathrm{~h}$, and had developed into vegetative bacteria by $4 \mathrm{~h}$.

${ }^{f} \mathrm{AA}$ refers to $\mathrm{L}$-alanine and $\mathrm{L}$-inosine (each at $10 \mathrm{mM}$, in $\mathrm{PBS} \mathrm{pH}$ 7.2).

L-inosine (each at $10 \mathrm{mM}$, in phosphate buffered saline (PBS) $\mathrm{pH}$ 7.2) (Table 1). In addition, the capacity of spore preparations to germinate and outgrow were confirmed by incubating dormant spores in the presence of Luria-Bertani broth (LB) (Table 1), as previously reported [41-43]. The time dependent increase in culture density (Figure 1A) and morphological conversion of spores into elongated bacilli (Figure 1C) indicated that in medium containing FBS, there was outgrowth of spores into vegetative bacilli.

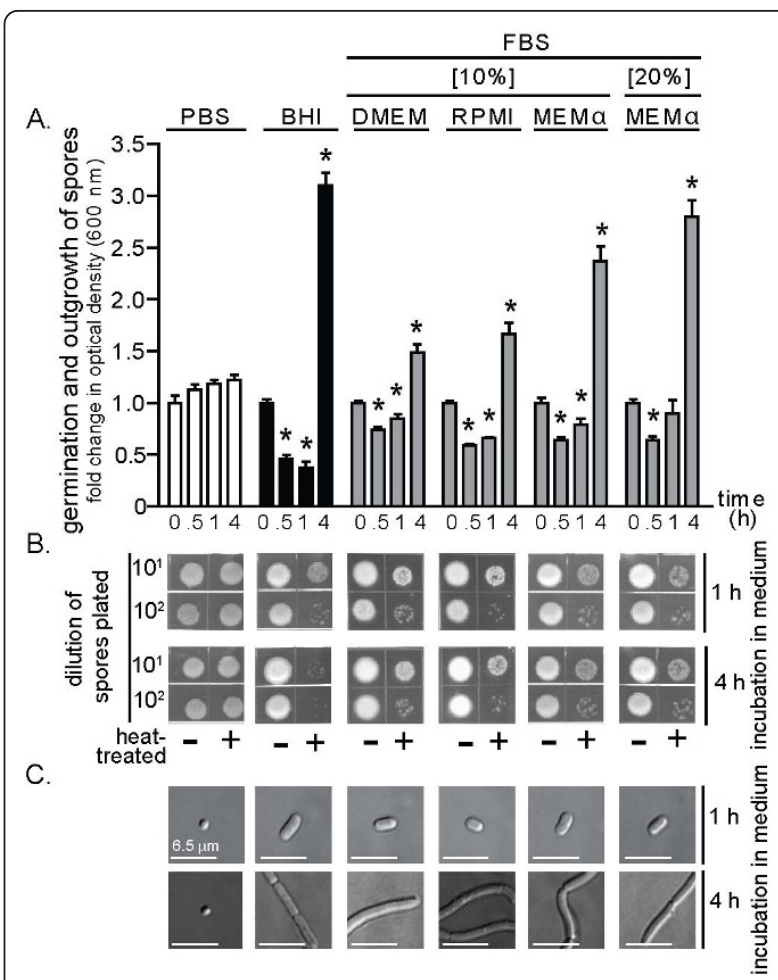

Figure $1 \mathrm{FBS}$ in cell culture media promotes germination and outgrowth of $B$. anthracis spores. B. anthracis spores were incubated in 96 -well plates at $37^{\circ} \mathrm{C}$ and with rotary agitation in the indicated medium. Germination and outgrowth of spores were monitored at the indicated times. Medium conditions are listed at the top of the figure, and applicable to (A-C). (A) Optical determination of germination and outgrowth. The data are rendered as the O.D.600 $\mathrm{nm}$ of the spore suspension at the indicated times relative to the original O.D.600 $\mathrm{nm}$ of the spore suspension at time $=0$ of the $37^{\circ} \mathrm{C}$ incubation. For BHI, DMEM, RPMI, and MEM $\alpha$, initial decreases in O.D.600 $\mathrm{nm}$ reflect the loss of spore refractility that occurs subsequent to germination initiation, while the increases in O.D.600 $\mathrm{nm}$ measured at later time points ( 1 and $4 \mathrm{~h}$ ) reflects bacterial replication. For PBS, the modest increases in O.D.600 nm are due to time-dependent medium evaporation. Error bars indicate standard deviations. For each medium tested, the $P$-values were calculated to evaluate the statistical significance of the differences between O.D. $600 \mathrm{~nm}$ values at the indicated times and O.D.600 nm values at the initial time point. (B) Spore heat sensitivity as a function of medium conditions. Aliquots from spore cultures were removed at indicated times, incubated for $30 \mathrm{~min}$ at either at $65^{\circ} \mathrm{C}$ or on ice, diluted $10^{1}$ - or $10^{2}$-fold (PBS pH 7.2), spotted (10 $\mu \mathrm{L}$ ) on LB plates, and incubated at $25^{\circ} \mathrm{C}$. After $18 \mathrm{~h}$, the plates were photographed. (C) Visual determination of $B$. anthracis spore outgrowth as a function of cell culture medium. Aliquots from spore cultures were removed at indicated times and analyzed for outgrowth using DIC microscopy. The bars indicate a length of 6.5 $\mu \mathrm{m}$. The data in (A) are combined from 3 independent experiments. The data in (B) and (C) are from a single experiment, and are representative of 3 independent experiments. 
In the absence of FBS, several media were discovered to induce germination initiation and outgrowth of B. anthracis spores (Table 1). Germination initiation (30-60 min) and outgrowth were detected when spores were incubated in brain heart infusion (BHI) broth (Table 1, Figure 2), modified minimum essential medium alpha modification (MEM $\alpha$ ) (Table 1, Figure 2), $\mathrm{CO}_{2}$-independent media (CIM) (Table 1), or McCoy's 5A (M5A) (Table 1). Each of these cell culture formulations contains all 20 amino acids, is enriched particularly in the known germinant L-alanine $(15-20 \mathrm{mg} / \mathrm{L})$, and also contains non-specified nucleotides. Notably, some nucleotides function as germinants $[35,44,45]$. In contrast, spores incubated in MEM, DMEM, RPMI 1640 medium, advanced MEM (AMEM), eagle MEM (EMEM), basal medium eagle (BME), or Ham's F-12 (F12) did not germinate, even after $4 \mathrm{~h}$ (Figure 2, Table 2). Each of these media possesses lower concentrations of L-alanine $(<10 \mathrm{mg} / \mathrm{L})$ than those media that induced germination, and generally lacked nucleotides. These results emphasize that care must be exercised when selecting a culture medium for conducting in vitro infections under non-germinating conditions.

\section{Effects of pre-conditioned culture medium on the germination state of $B$. anthracis spores}

We next considered the possibility that cell culture media that normally do not promote spore germination may be converted to germinating media when incubated in the presence of mammalian cells. To evaluate this possibility, B. anthracis spores were incubated in DMEM or RPMI that had been "pre-conditioned" in the presence of RAW264.7 cells or MH-S cells, respectively. These studies revealed that neither DMEM nor

Table 2 Germination and outgrowth of B. anthracis spores as a function of FBS concentration ${ }^{a}$

\begin{tabular}{ccccc}
\hline & & & \multicolumn{2}{c}{ outgrowth $^{\boldsymbol{e}}$} \\
\cline { 4 - 5 } medium $^{b}$ & FBS (\%) & germination $^{\boldsymbol{c}}$ & $\mathbf{1 ~ \mathbf { ~ }}$ & $\mathbf{4} \mathbf{~ h}$ \\
\hline DMEM & 0.0 & - & - & - \\
& 0.1 & - & - & - \\
& 0.5 & - & - & - \\
& 1.0 & + & - & + \\
& 5.0 & + & + & + \\
& 10.0 & + & + & + \\
\hline
\end{tabular}

\footnotetext{
${ }^{a}$ Three independent experiments were performed with three different spore preparations, each conducted in triplicate.

${ }^{b}$ Spores prepared from B. anthracis Sterne 7702 were incubated in DMEM.

${ }^{c}$ Indicates the concentration of FBS used in the DMEM.

${ }^{d}$ Spores were scored positive (+) for germination if the $\mathrm{OD}_{600 \mathrm{~nm}}$ of the suspended spores decreased by more than $5 \%$ after 30 min incubation in the indicated medium.

${ }^{e}$ Using DIC microscopy, spores were scored positive (+) for outgrowth if the spores bodies were visibly larger at $1 \mathrm{~h}$, and had developed into vegetative bacteria by $4 \mathrm{~h}$.
}

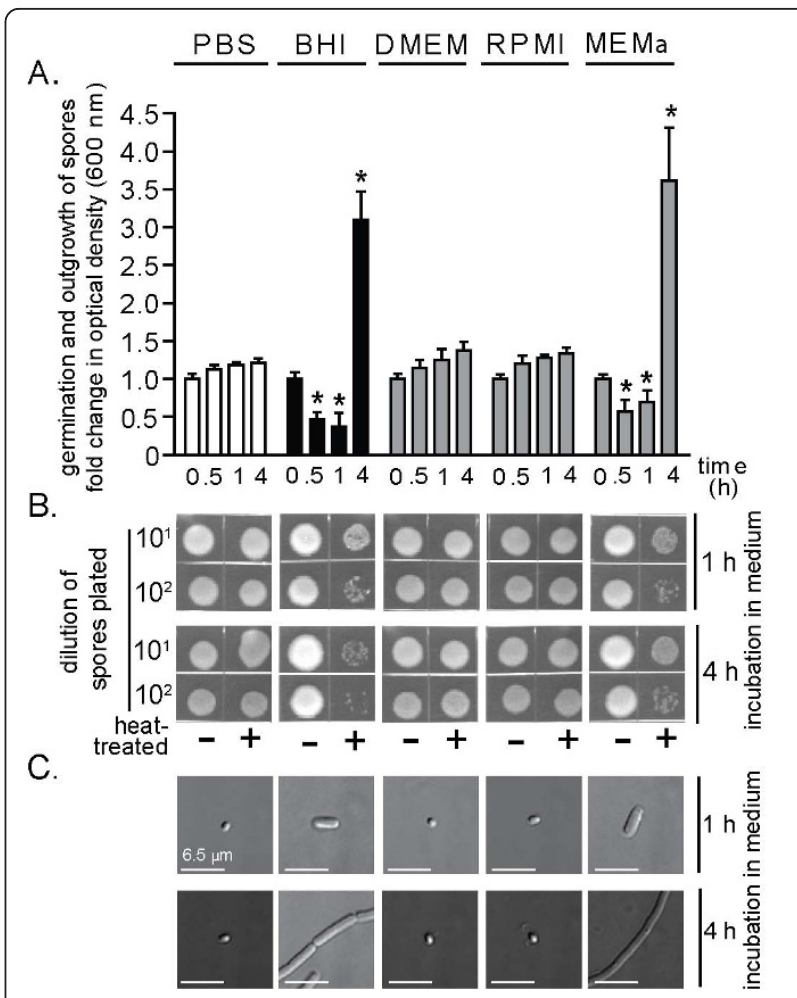

Figure 2 B. anthracis spore germination and outgrowth in FBSfree cell culture media. B. anthracis spores were incubated in 96well plates at $37^{\circ} \mathrm{C}$ and with rotary agitation within the indicated medium. Germination and outgrowth of spores were monitored at the indicated times. Medium conditions are listed at the top of the figure, and are applicable to (A-C). (A) Optical determination of germination and outgrowth. The data are rendered as the O.D.600 $\mathrm{nm}$ of the spore suspension at the indicated times relative to the original O.D.600 nm of the spore suspension at time $=0$ of the $37^{\circ} \mathrm{C}$ incubation. Error bars indicate standard deviations. For each medium tested, the $P$-values were calculated to evaluate the statistical significance of the differences between O.D. $600 \mathrm{~nm}$ values at the indicated times and O.D.600 nm values at the initial time point. (B) Spores heat sensitivity as a function of medium conditions. Aliquots from the spore cultures were removed at indicated times, incubated for $30 \mathrm{~min}$ at either at $65^{\circ} \mathrm{C}$ or on ice, diluted $10^{1}$ - or $10^{2}$-fold (PBS pH 7.2), spotted (10 $\left.\mu \mathrm{L}\right)$ on LB plates, and incubated at $25^{\circ} \mathrm{C}$. After $18 \mathrm{~h}$, the plates were photographed. (C) Visual determination of $B$. anthracis spore outgrowth as a function of cell culture medium. Aliquots from the spore cultures were removed at indicated times and analyzed for outgrowth using DIC microscopy. The bars indicate a length of $6.5 \mu \mathrm{m}$. The data in (A) are combined from 3 independent experiments. The data in (B) and $(C)$ are from a single experiment and are representative of 3 independent experiments.

RPMI, following a pre-conditioning period of $4 \mathrm{~h}$, induced germination of $B$. anthracis spores (Figure 3A). Likewise, medium withdrawn from RAW264.7 cells infected for 1 or $4 \mathrm{~h}$ with dormant spores at a multiplicity of infection of 10 (MOI 10) also remained non-germinating (Figure 3B). Finally, medium withdrawn from RAW264.7 cells infected with dormant 
A.

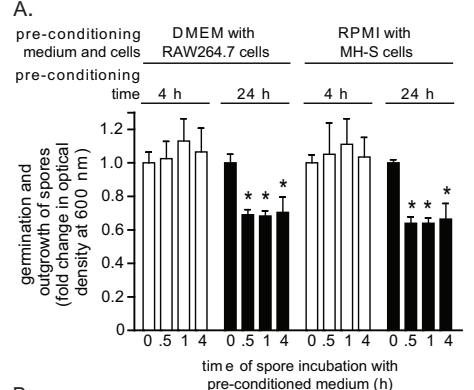

B.

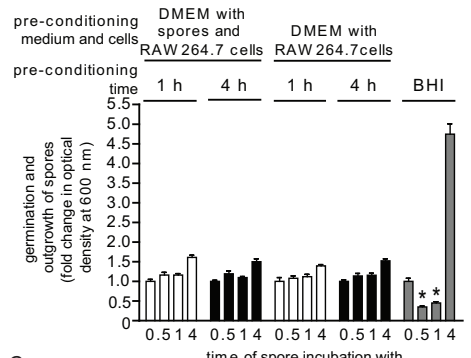

C. time of spore incubation with
pre-conditioned medium $(\mathrm{h})$

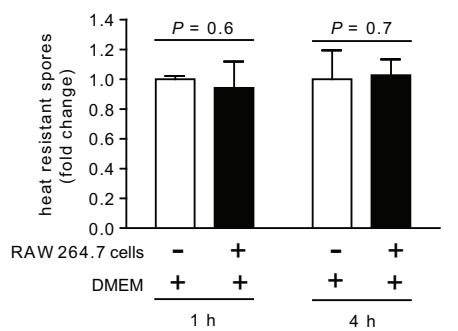

Figure 3 The effects of pre-conditioned culture medium on the germination state of $B$. anthracis spores. DMEM (A, B) or RPMI (B) were pre-conditioned by incubating with monolayers of RAW264.7 $(\mathrm{A}, \mathrm{B})$ or $\mathrm{MH}-\mathrm{S}$ cells $(\mathrm{B})$ at $37^{\circ} \mathrm{C}$ and under $5 \% \mathrm{CO}_{2}$, in the absence (A) or presence (MOI 10) (B) of B. anthracis spores. (A, B). After $4 \mathrm{~h}$ (white bars) or $24 \mathrm{~h}$ (black bars) (A), or after 1 (white bars) and $4 \mathrm{~h}$ (black bars) (B), the medium was removed from the monolayers, filter sterilized, and then incubated with B. anthracis spores in 96well plates at $37^{\circ} \mathrm{C}$ and with rotary agitation. Germination and outgrowth of spores were monitored at indicated times by measuring $0 . D_{.600} \mathrm{~nm}$. The results are rendered as the O.D.600 nm of the spore suspension at the indicated time relative to the original O.D.600 nm of the spore suspension at time $=0$ of the $37^{\circ} \mathrm{C}$ incubation. P-values were calculated to evaluate the statistical significance of the differences between O.D.600 nm values at the initial time point and O.D. O.D.600 nm values at the indicated times. For (B), BHI (gray bars) was used as a positive control for germination and outgrowth. (C) An equal number of $B$. anthracis spores were incubated at $37^{\circ} \mathrm{C}$ and under $5 \% \mathrm{CO}_{2}$ in DMEM (no FBS) in the absence (white bars) or presence (black bars) of RAW264.7 cells (MOI 10). At indicated times, aliquots of culture medium were removed, and spores were evaluated for heat resistance. The results are rendered as the number of heat resistant spores relative to spores incubated in DMEM alone, which were normalized to 1.0. P-values were calculated to evaluate the statistical significance of the differences in heat resistant spores between those incubated in the presence or absence of RAW264.7 cells. The data in $(A-C)$ are combined from 3 independent experiments conducted in triplicate with error bars indicating standard deviations. spores (MOI 10) contained only heat resistant $B$. anthracis, and no heat sensitive spores (Figure 3C), indicating that the extracellular spores remained dormant through the first 4 hours of infection. When the pre-conditioning period was extended to $24 \mathrm{~h}$, both DMEM and RPMI induced germination, but negligible outgrowth, of spores (Figure 3A). Spore germination was eliminated by dialyzing (12-14 kDa molecular mass cutoff) the $24 \mathrm{~h}$ preconditioned DMEM or RPMI, but not by heat treatment $\left(95^{\circ} \mathrm{C}\right.$ for $10 \mathrm{~min}$, or, $65^{\circ} \mathrm{C}$ for $30 \mathrm{~min}$; data not shown), suggesting that the germinating factors were relatively small molecular weight, heat-resistant factors. Nonetheless, these studies confirm that in vitro models can be established that maintain a non-germinating environment for at least the first $4 \mathrm{~h}$ of infection.

Mammalian cells remain viable and functional for at least $4 \mathrm{~h}$ in FBS-free culture medium

Although a non-germinating environment was maintained for at least $4 \mathrm{~h}$ in FBS-free media (Figure 3), it was unclear whether viable and functional cells could be maintained in FBS-free medium over this same time period. Studies to evaluate this issue revealed that over a $4 \mathrm{~h}$ period, RAW264.7 cells in DMEM demonstrated essentially identical viability (Figure 4A), cell cycle progression (Figure 4B), and metabolic activity (Figure $4 \mathrm{C}$ ) in the absence or presence of FBS (10\%). Even after 24 $\mathrm{h}$, the viability (Figure $4 \mathrm{~A}$ ) and cell cycle profiles (Figure 4B) were not significantly different for RAW264.7 cells cultured in the absence or presence of FBS. The metabolic activity of RAW264.7 cells increased after $24 \mathrm{~h}$, but significantly more so in the presence than absence of FBS (Figure 4C), which we speculate was due to greater overall proliferation and number of cells in FBSenriched medium. These results confirmed that, for at least $4 \mathrm{~h}$, in vitro models of infection can be conducted under entirely non-germinating culture conditions without loss of host cell viability, cell cycle progression, or metabolic function.

\section{Germination state of spores does not alter the uptake by mammalian cells}

The demonstration that cultured RAW264.7 cells remained viable and functional in FBS-free cell culture medium did not directly address the possibility that spore uptake by mammalian cells might be substantially different under germinating and non-germinating cell culture conditions. To evaluate this issue, Alexa Fluor 488-labeled spores were incubated with RAW264.7, $\mathrm{MH}-\mathrm{S}$, or JAWSII cells (MOI 10) in the absence or presence of FBS (10\%). After 5 or $60 \mathrm{~min}$, intracellular spores were monitored using flow cytometry to measure cell associated fluorescence that was not sensitive to the 


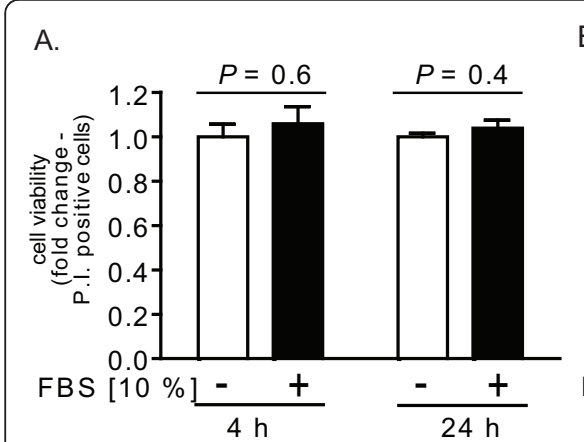

B.

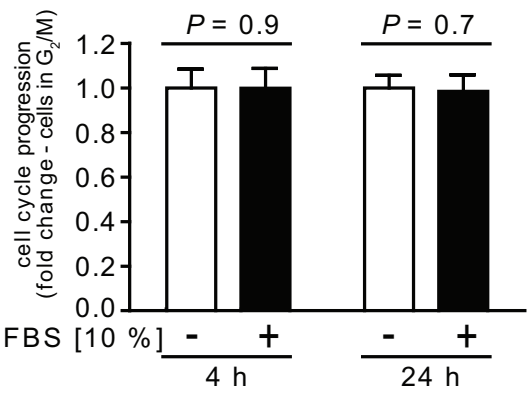

C.

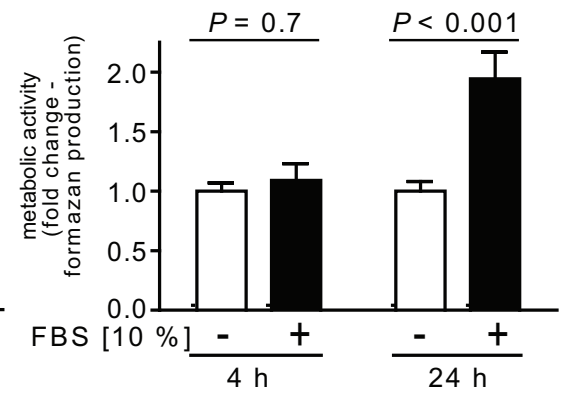

Figure 4 Effect of non-germinating conditions on RAW264.7 cell viability, cell cycle progression, and metabolic activity. RAW264.7 cells were incubated at $37^{\circ}$ in DMEM in the presence (+, black bars) or absence (-, white bars) of FBS, and then evaluated at 4 or $24 \mathrm{~h}$, as indicated, for viability (A), cell cycle progression (B), and metabolic activity (C). (A) The cells were assayed for PI uptake, as described under Materials and Methods. The data are rendered as the relative PI uptake normalized at both 4 and $24 \mathrm{~h}$ to cells incubated in the absence of FBS. (B) The cells were analyzed for their cell cycle profiles, as described under Materials and Methods. The data are rendered as the relative numbers of cells in $\mathrm{G}_{2} / \mathrm{M}$ normalized at both 4 and $24 \mathrm{~h}$ to cells incubated in the absence of FBS. (C) The cells were analyzed for conversion of MTT to formazan. The data are rendered as the fold change of formazan production normalized at both 4 and $24 \mathrm{~h}$ to cells incubated in the absence of FBS. To generate the bar graphs, data were combined from three independent experiments, each conducted in triplicate. Error bars indicate standard deviations. The $P$ values were calculated to evaluate the statistical significance of the differences in viability (A), cell cycle progression (B), and metabolism (C) between cells cultured in the absence or presence of FBS.

membrane-impermeable, Alexa Fluor 488 quenching agent, trypan blue [46]. These studies revealed that for each cell line tested, neither the percentage of infected cells within the population (Figure 5A-C), nor the overall increase in intracellular spores (Figure 5D-F), was significantly different in the presence or the absence of FBS. Collectively, these results revealed that the uptake of $B$. anthracis spores by mammalian cells is essentially the same within germinating and non-germinating in vitro environments.

\section{Germination state of spores influences the number of viable, intracellular $B$. anthracis}

Although the uptake of $B$. anthracis spores into mammalian cells was independent of the presence or absence of FBS in the culture medium, it was not clear whether the outcome of infection would also be similar under germinating and non-germinating conditions. To evaluate this issue, the recovery of viable, intracellular $B$. anthracis was compared subsequent to uptake by RAW264.7 cells in the absence or presence of FBS (10\%), using the gentamicin protection assay $[11,21,46,47]$. These studies indicated that there were not significant differences in intracellular CFU after 5 min post-infection (Figure 6). However, after 60 or 240 min post infection, significantly greater CFU were recovered from cells in DMEM lacking FBS relative to cells incubated in the presence of FBS (Figure 6). To evaluate whether these differences might be attributed strictly to the presence or absence of FBS, similar studies were conducted in the absence of FBS, however this time using spores that had been pre-germinated for $30 \mathrm{~min}$ with DMEM supplemented with L-alanine/L-inosine (both at $10 \mathrm{mM}$ ). Similar to spore uptake in the presence of FBS, significantly fewer CFU were recovered from cells incubated with pre-germinated spores in the absence of FBS relative to cells incubated with dormant spores in DMEM lacking FBS (Figure 6). These results support the idea that the germination state of spores, rather than the presence or absence of FBS, is an important determinant of the outcome of infection. Similar results were obtained when studies were conducted with $\mathrm{MH}-\mathrm{S}$ cells and JAWSII cells (not shown). Although the reasons underlying the greater recovery of spores from infections conducted under non-germinating conditions are not clear, we speculate that germinated spores might be more susceptible than dormant spores to killing after uptake from the cell surface. This potential explanation is consistent with earlier reports that spores that had been intentionally pre-germinated prior to exposure to mammalian cells were more readily killed than dormant spores upon uptake into mammalian cells $[20,22]$. These results support the idea that the germination state of $B$. anthracis spores is a critical determinant of the fate of the intracellular bacteria.

\section{Germination state of $B$. anthracis spores influences the viability of RAW264.7 cells during in vitro infection} The greater number of viable, intracellular B. anthracis recovered from cells infected under non-germinating conditions (Figure 6) prompted us to examine whether the viability of infected host cells might also be influenced by the germination state of spores during uptake. To evaluate this issue, RAW264.7 cells were incubated 


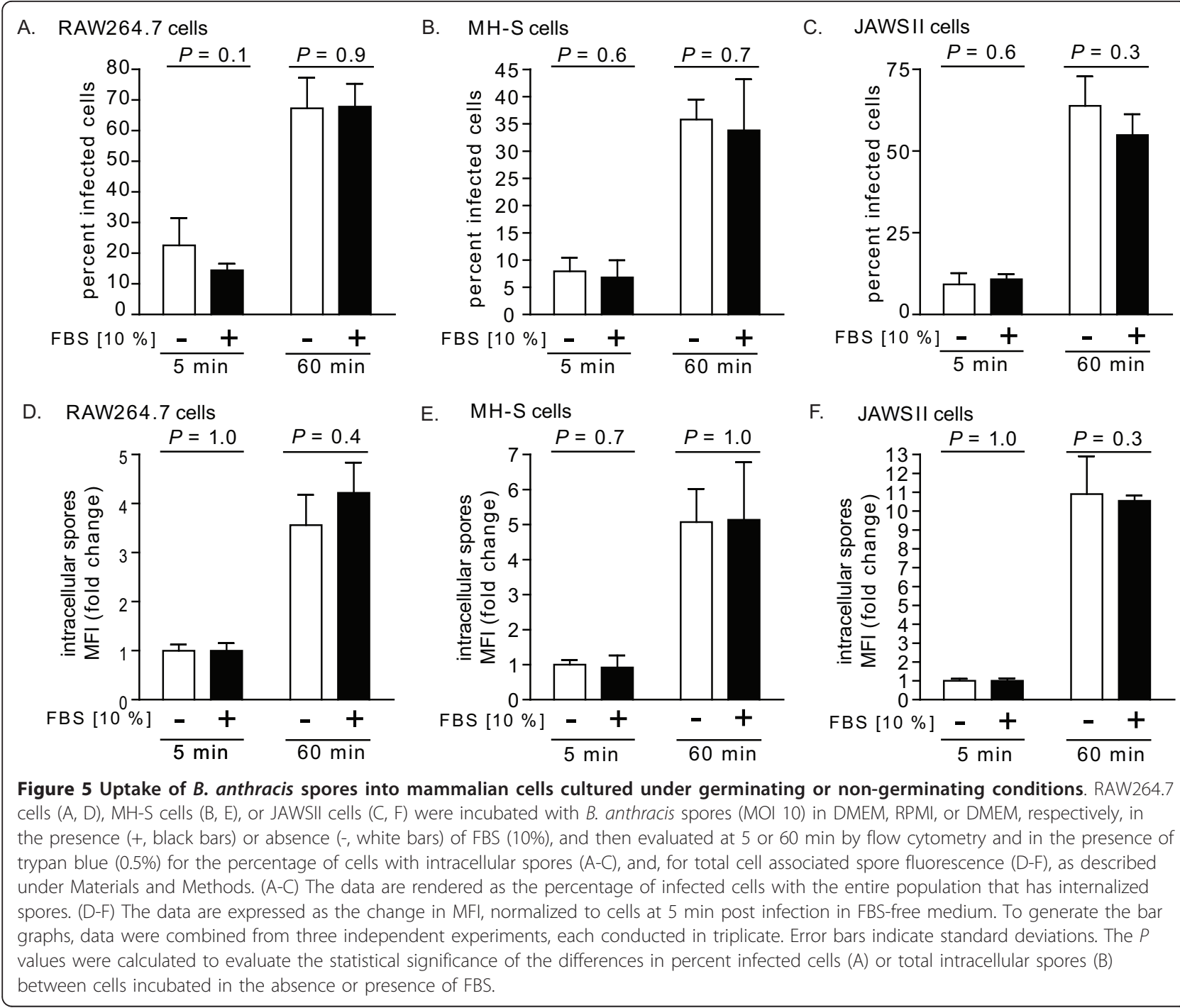

with B. anthracis spores (MOI 10) in the presence or absence of FBS (10\%). Subsequent to employing the same gentamicin-protection procedure used for monitoring intracellular B. anthracis (Figure 6), PI uptake by RAW264.7 cells was measured at $5 \mathrm{~min}, 1 \mathrm{~h}$, and $4 \mathrm{~h}$, post-infection. These studies revealed that at $4 \mathrm{~h}$ postinfection, there was approximately 2 -fold greater PI uptake, indicating a significantly greater loss in viability of RAW264.7 cells that had been incubated with spores in FBS-deficient medium, as compared to FBS-enriched medium (Figure 7 ). When evaluated at $8 \mathrm{~h}$ post-infection, PI uptake was nearly 5-fold greater in RAW264.7 cells that had been incubated with $B$. anthracis spores in FBS-deficient medium (data not shown). Understanding the reasons underlying these significant differences in the viability of infected cells will require future studies, but we speculate that the greater intracellular load of $B$. anthracis in cells infected under non-germinating conditions (Figure 6) may directly contribute to the higher degree of cell death.

\section{The importance of culture medium during in vitro infection models}

Despite compelling evidence that during in vivo infection, the alveolar spaces of the lungs are intrinsically non-germinating, and dormant spores are taken up by mammalian cells prior to germination [5-7,23-27], many studies involving in vitro models of infection have been conducted under germinating medium conditions [20,28-34]. Most studies have been conducted in cell culture medium containing 2-10\% FBS, including those using RAW264.7 cells [48,49], and the germination state of spores have not generally monitored or controlled for during in vitro infections. 


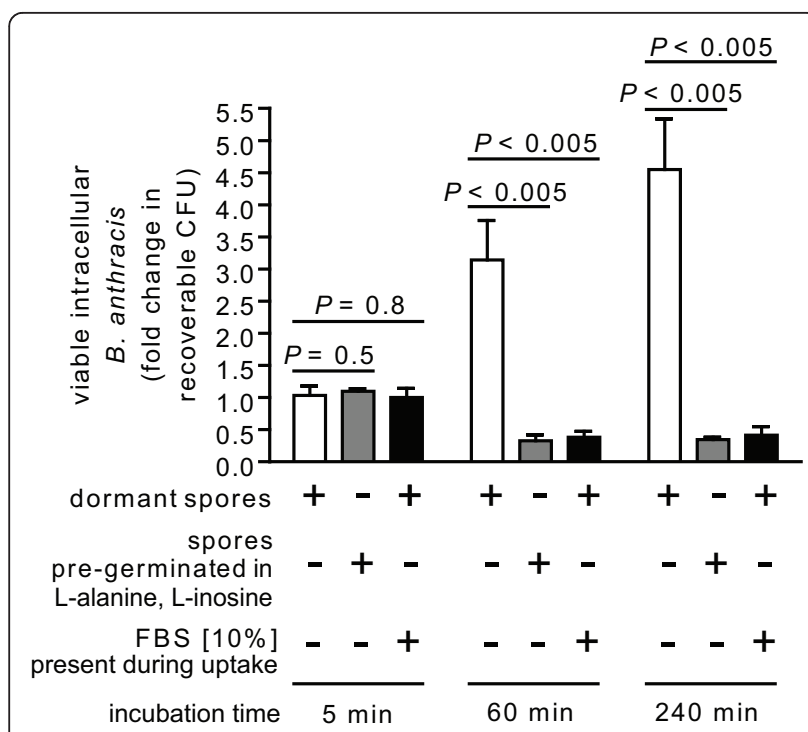

Figure 6 The germination state of spores influences the viability of intracellular $\boldsymbol{B}$. anthracis. RAW264.7 cells were incubated for 30 min with dormant $B$. anthracis spores (MOI 10) in DMEM in the presence $(+$, black bars) or absence $(-$, white bars) of FBS (10\%), or, with pre-germinated spores (MOI 10) in DMEM in the absence of FBS (grey bars). B. anthracis spores were pre-germinated by incubation for 30 min in PBS pH 7.2 supplemented with Lalanine and L-inosine (both at $10 \mathrm{mM}$ ), and then washed twice with PBS pH 7.2 to remove germinants. After $30 \mathrm{~min}$, the cells were washed to remove extracellular $B$. anthracis, and then further incubated with FBS (10\%) and, as described under "Methods," with gentamicin to germinate and kill any remaining spores that had not been germinated. After $15 \mathrm{~min}$, the cells were washed and then further incubated in the absence of gentamicin. At 5, 60, or $240 \mathrm{~min}$ after removal of gentamicin, as indicated, the RAW264.7 cells were lysed, and the lysates were evaluated for viable B. anthracis, as described under Materials and Methods. The data were rendered as the fold-change in recoverable CFU in the absence or presence of FBS, relative to cells at 5 min post infection in the absence of FBS. The rendered data were combined from three independent experiments, each conducted in triplicate. Error bars indicate standard deviations. The $P$ values were calculated to evaluate the statistical significance of the differences in recoverable CFU between cells infected in the absence or presence of FBS.

Several in vitro models have employed additives to the culture medium in an attempt to modulate germination. Several studies used D-alanine and/or D-histidine $[22,32,50]$, known inhibitors of germination initiation. However, interpretation of these studies may be complicated by the finding that $\mathrm{D}$-alanine/D-histidine, when added subsequent to spore uptake into macrophages, alter the extent to which spores germinate [32], suggesting that these D-amino acid germination inhibitors diffuse into host cells and affect spore germination within intracellular vesicles. Horse serum has been used by several groups to limit spore outgrowth during infection [20,32,33,51]. However, $10 \%$ horse serum in DMEM only slows, but does not eliminate the germination initiation of spores [20]. The finding that RAW264.7 cells maintain viability, cell cycle progression, and mitochondrial metabolic activity for at least $4 \mathrm{~h}$ when maintained in serum-free medium (Figure 4), indicate that in vitro infections, at least with RAW 264.7 cells, can be conducted under non-germinating conditions using FBS-free medium.

\section{The outcome of infection is influenced by the germination state of spores}

Both spore (Figure 6) and host cell (Figure 7) viability were influenced by the germination state of spores at the time of uptake. Because several cell lines internalized the same number of spores under both germinating and non-germinating conditions (Figure 5), it is unlikely that differences in the outcome of infection are due solely to initial differences in spore load. Rather, we speculate that, in contrast to dormant spores, germinated spores might be more vulnerable to growth inhibition and/or killing during phagocytosis. These results are consistent with previous reports that when infections were conducted with spores in medium containing FBS or fetal calf serum (e.g. germinating conditions), there were generally, within the first 4-5 $\mathrm{h}$

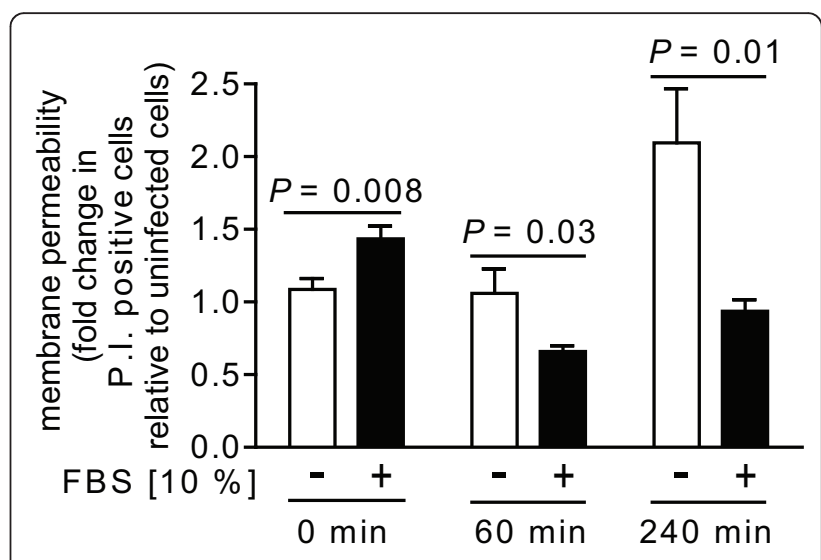

Figure 7 The germination state of spores influences the viability of B. anthracis-infected cells. RAW264.7 cells were incubated for 30 min with $B$. anthracis spores ( $\mathrm{MOI} 10$ ) in DMEM in the presence (+, black bars) or absence (-, white bars) of FBS $(10 \%)$. After $30 \mathrm{~min}$, the cells were washed to remove extracellular $B$. anthracis, and then further incubated with FBS (10\%) and, as described under "Methods," with gentamicin to germinate and kill any remaining spores that had not been germinated. After $15 \mathrm{~min}$, the cells were washed and then further incubated in the absence of gentamicin. At 0 (immediately after gentamicin removal), 60, or 240 min after removal of gentamicin, as indicated, the cells were evaluated for mammalian cell death via PI uptake, as described under Materials and Methods. The data are rendered as the foldincrease of PI uptake relative to non-infected cells in the absence or presence of FBS at 5, 60, or $240 \mathrm{~min}$, as indicated. The rendered data have been combined from three independent experiments, each conducted in triplicate. Error bars indicate standard deviations. The $P$ values were calculated to evaluate the statistical significance of the differences between the fold-increase of PI uptake between cells incubated with spores in the absence or presence of FBS. 
post-infection, losses in intracellular CFU recovered from primary human dendritic cells [17], primary mouse alveolar macrophages [17], J774.A1 murine macrophage-like cells [18], bone marrow derived macrophages from A/J mice [34], or RAW 264.7 cells [13].

\section{Conclusions}

This study demonstrates that the infection of RAW 264.7 cells by $B$. anthracis spores is influenced by the germination state of spores, as dictated by the in vitro culture medium. The extent to which the germination state of $B$. anthracis spores ultimately affects the outcome of infections using cells other than RAW264.7 cells may ultimately depend on the properties idiosyncratic to that particular cell type or cell line. However, our results indicate the importance of rigorously considering the germinating properties of the culture medium when establishing in vitro models to study the infection of host cells with $B$. anthracis spores.

\section{Methods}

\section{Spore preparations and fluorescent labeling}

Spores were prepared from B. anthracis Sterne 7702 and enumerated using a hemacytometer (Thermo Fisher Scientific, Waltham, MA), as described previously [46]. As quality control, spore preparations were tested for both heat resistance and the capacity to germinate, as described [46].

\section{Mammalian cell culture}

Abelson murine leukemia virus-transformed murine macrophages derived from ascites of BALB/c mice (RAW 264.7 macrophage-like cells; CRL-2278; ATCC, Manassas, VA) were maintained within a humidified environment at $37^{\circ} \mathrm{C}$ and under $5 \% \mathrm{CO}_{2}$ in complete DMEM, (Thermo Scientific, Waltham, MA) containing penicillin (100 U; Gibco BRL, Grand Island, NY), streptomycin $(0.1 \mathrm{mg} / \mathrm{ml}$; Gibco BRL), L-glutamine (2 mM; Sigma, St. Louis, MO), and FBS (10\%; JRH Biosciences, Lenexa, KS). MH-S cells (CRL-2019; ATCC) were maintained within a humidified environment at $37^{\circ} \mathrm{C}$ and under $5 \% \mathrm{CO}_{2}$ in complete RPMI medium (Thermo Scientific) containing penicillin-streptomycin (100 U, Gibco BRL), L-glutamine (4 mM), and FBS (10\%). JAWSII (CRL-11904; ATCC) were maintained within a humidified environment at $37^{\circ} \mathrm{C}$ and under $5 \% \mathrm{CO}_{2}$ in complete MEM $\alpha$ (Thermo Scientific) containing penicillin-streptomycin (100 U), L-glutamine (4 mM), and FBS (20\%). All tissue culture plasticware was purchased from Corning Incorporated (Corning, NY).

\section{Evaluation of $B$. anthracis spore germination in cell culture media}

Using 96 well plates, spores prepared from $B$. anthracis $7702\left(1.0 \times 10^{8}\right.$ spores $\left./ \mathrm{mL}\right)$ were incubated at $37^{\circ} \mathrm{C}$ and under $5 \% \mathrm{CO}_{2}$ in BHI (BD Biosciences, San Jose, CA), LB $(0.1 \%$ tryptone, BD Biosciences; $0.05 \%$ yeast extract, BD Biosciences; 0.05\% NaCl, Fisher Chemical, Fairlawn, NJ), PBS pH 7.2 (Mediatech, Manassas, VA), or germinating amino acids (10 mM L-alanine, $10 \mathrm{mM}$ L-inosine, both from Sigma) in PBS pH 7.2. In other studies, spores were incubated in 96 well plates $\left(10^{8}\right.$ spores $\left./ \mathrm{mL}\right)$ and at $37^{\circ} \mathrm{C}$ and under $5 \% \mathrm{CO}_{2}$ in the following cell culture media without or with FBS (10\%, unless otherwise indicated; Mediatech): $\operatorname{DMEM}(0.1,0.5,1,5$ or $10 \%$ FBS), RPMI-1640, MEM $\alpha$ modification (10 or $20 \%$ FBS), MEM (Mediatech), AMEM (Gibco), EMEM (Mediatech), BME (Sigma), CIM (Gibco), Ham's F-12 (Mediatech), McCoy's 5A (M5A, ATCC), or DMEM with 10\% FBS and $10 \mathrm{mM}$ D-alanine (Sigma) and D-histidine (Sigma). In some assays, FBS obtained from Mediatech was substituted with FBS purchased from Invitrogen or Sigma. As described previously [39], spore germination was evaluated by measuring loss in spore refractility or loss of heat resistance, while outgrowth was monitored by monitoring the elongation of bacilli using a Delta Vision RT microscope (Applied Precision; Issaquah, WA), outfitted with an Olympus Plan Apo $100 \times$ oil objective. DIC images were collected using a Photometrics CoolSnap HQ camera; (Photometrics, Tucson; AZ), and processed using SoftWoRX Explorer Suite (version 3.5.1, Applied Precision Inc).

\section{Pre-conditioning of cell culture media}

To pre-condition cell culture medium, monolayers of RAW264.7 or MH-S cells in 24-well plates (80 to $95 \%$ confluency) were washed three times with Hanks' balanced salt solution (HBSS) and then incubated in DMEM (for RAW264.7 cells) or RPMI-1640 (for MH-S cells) without FBS and penicillin-streptomycin in a humidified environment at $37^{\circ} \mathrm{C}$ and under $5 \% \mathrm{CO}_{2}$. After 4 or $24 \mathrm{~h}$, the medium was withdrawn, centrifuged $(600 \times g$ for $5 \mathrm{~min})$, and the supernatant was filter sterilized using a $0.22 \mu \mathrm{m}$ filter (Corning). To evaluate heat sensitivity, some of the filter-sterilized pre-conditioned medium was incubated at $95^{\circ} \mathrm{C}$ for $10 \mathrm{~min}$ or, alternatively, $65^{\circ} \mathrm{C}$ for $30 \mathrm{~min}$ Alternatively, some of the filtersterilized pre-conditioned medium $(3 \mathrm{~mL})$ was dialyzed four times against PBS pH $7.2(500 \mathrm{~mL})$, using dialysis tubing with 12,000-14,000 molecular mass cutoff (Spectrum Laboratories, Inc., Rancho Dominguez, CA), each time for $6 \mathrm{~h}$.

\section{Mammalian cell viability}

To evaluate the viability of RAW264.7, MH-S, or JAWSII cells, alterations in membrane permeability, as indicated by relative PI $(1 \mu \mathrm{g} / \mathrm{mL}$; Invitrogen Molecular Probes, Eugene, OR) uptake, were measured using flow cytometry, as previously described [46]. 


\section{Flow cytometry}

Analytical flow cytometry was carried out using a Beckman Coulter EPICS XL-MCL ${ }^{\mathrm{TM}}$ flow cytometer equipped with a 70- $\mu \mathrm{m}$ nozzle, $488 \mathrm{~nm}$ line of an aircooled argon-ion laser, and $400 \mathrm{mV}$ output. The band pass filter used for detection of Alexa Fluor 488 spores was 525/10 nm. The long pass filter used for cell cycle phase determination assays and mammalian cell viability assays was $655 \mathrm{~nm} / \mathrm{LP}$. Cell analysis was standardized for side/forward scatter and fluorescence by using a suspension of fluorescent beads (Beckman Coulter Inc., Fullerton, CA). At least 10,000 events were detected for each experiment ( $>2000$ events per min). Events were recorded on a log fluorescence scale and evaluated using FCS Express 3.00.0311 V Lite Standalone. Sample debris (as indicated by lower forward and side scatter and a lack of PI staining) represented a small fraction (1 to $2 \%)$ of the detected events and was excluded from analysis.

\section{Cell cycle assay}

To compare the cell-cycle profiles of RAW264.7 cells cultured in FBS-containing medium or FBS-free medium, relative PI uptake was measured using flow cytometry. At 4 or $24 \mathrm{~h}$, as indicated, cells were incubated at room temperature with Cellstripper ${ }^{\mathrm{TM}}$ (Mediatech). After $15 \mathrm{~min}$, the cells were further diluted with PBS pH 7.2 containing $10 \%$ FBS $(800 \mathrm{~mL})$. The cell suspensions were centrifuged for $5 \mathrm{~min}$ at $500 \times \mathrm{g}$ at room temperature. The pellets were resuspended in $300 \mu \mathrm{L}$ of PBS pH 7.2 at room temperature, fixed by adding anhydrous ethanol $\left(100 \%, 700 \mu \mathrm{L}\right.$ prechilled to $-20^{\circ} \mathrm{C}$, Fisher Scientific) with continuous vortexing, and then further incubated for at least $2 \mathrm{~h}$ at $-20^{\circ} \mathrm{C}$. The cells were centrifuged for $5 \mathrm{~min}$ at $500 \times g$ at room temperature, and the pellets were resuspended in $1 \mathrm{~mL}$ of PBS pH 7.2, and then incubated at room temperature for $30 \mathrm{~min}$. The cells were centrifuged $5 \mathrm{~min}$ at $500 \times \mathrm{g}$ at room temperature. The cell pellets were resuspended in 300 $\mu \mathrm{L}$ PBS pH 7.2, 0.1\% Triton X-100 (MP Biomedicals, Solon, OH), DNase-free RNase A (100 mg/mL; Sigma), and PI $(10 \mu \mathrm{g} / \mathrm{mL})$, and further incubated at room temperature for $60 \mathrm{~min}$. The stained cells were analyzed by flow cytometry.

\section{Mammalian cell metabolism assay}

To compare the metabolic activities of RAW264.7 cells cultured in FBS-containing medium or FBS-free medium, the relative conversion of tetrazolium 3-(4,5-dimethylthiazol-2-yl)-2,5-diphenyltetrazolium bromide (tetrazolium; $5 \mathrm{mg} / \mathrm{mL}$, Sigma) to formazan over $30 \mathrm{~min}$ and at $37^{\circ} \mathrm{C}$ was measured at $570 \mathrm{~nm}$ with a Synergy 2 plate reader (BioTek Instruments, Inc., Winooski, VT), as described $[39,52]$.

\section{In vitro infection of mammalian cells with $B$. anthracis}

Mammalian cells $\left(5.0 \times 10^{5}\right.$ total cells/well $)$ were incubated in the appropriate complete medium, as indicated above under "Mammalian cell culture," for two days in a humidified environment at $37^{\circ} \mathrm{C}$ and under $5 \% \mathrm{CO}_{2}$, resulting in $80-95 \%$ confluency. To calculate the number of spores needed to achieve MOI 10, cells from several wells were detached using Cellstripper ${ }^{\mathrm{TM}}$ and enumerated using a hemacytometer. The cells were used only if greater than $90 \%$ of the cells excluded trypan blue; generally, greater than $95 \%$ of the cells within the monolayer excluded trypan blue. Prior to the addition of labeled spores, cells were washed three times with HBSS and then incubated in DMEM (RAW264.7 and JAWSII) or RPMI-1640 (MH-S), without or with FBS, as indicated. To synchronize the exposure of cells to spores, spores were immediately and gently centrifuged $(600 \times g$ for $5 \mathrm{~min}$ ) onto the surfaces of cells. The plates were incubated within a humidified environment at $37^{\circ} \mathrm{C}$ and under $5 \% \mathrm{CO}_{2}$ for the indicated times prior to analysis.

\section{Quantification of B. anthracis uptake by mammalian cells}

Internalization of $B$. anthracis spores by mammalian cells was quantified using a previously described flow cytometry based assay [46]. Briefly, the indicated mammalian cell lines were seeded into 48-well plates (Corning) in order to achieve $80-95 \%$ confluency after two days of incubation. As previously described [46], $B$. anthracis spores were labeled using an amine reactive Alexa Fluor ${ }^{\circledR} 488$ carboxylic acid, succinimidyl ester (Molecular Probes-Invitrogen). Alexa Fluor 488-labeled B. anthracis spores were quantified using a hemacytometer, added to cells at the desired MOI, and immediately but gently centrifuged (300 $x g$ for $5 \mathrm{~min}$ ) onto the surface of cells. The plates were incubated within a humidified environment at $37^{\circ} \mathrm{C}$ and under $5 \% \mathrm{CO}_{2}$ for the indicated times prior to analysis using flow cytometry, as previously described [46]

To discriminate intracellular spores from those which remain surface-associated during infection, cells were analyzed in the presence of trypan blue, a membraneimpermeable, Alexa Fluor $488^{\circledR}$ fluorescence quenching agent [53]. Previously, 0.5\% trypan blue was demonstrated to completely quench the fluorescence emission of Alexa Fluor 488-labeled spores bound to the surface of mammalian cells, while having no affect the fluorescence emission of internalized spores [46]. From these data, the percentage of cells with internalized B. anthracis was calculated by dividing the number of viable cells with greater than background auto-fluorescence by the total number of viable cells. For spore internalization experiments, viable mammalian cells (typically 90-98\% of the total events) were readily identified by their high forward scatter and lack of propidium iodide (PI) 
staining. A second distinct population, (2-10\%) of dead cells was routinely detected with relatively lower forward scatter (which indicates a smaller size) and positive PI staining (indicating non-viable cells; data not shown). Over the course of $60 \mathrm{~min}$, we observed no detectable increase in cell death in the presence of labeled spores, as indicated by PI uptake (data not shown). Finally, sample debris (as indicated by relatively lower forward and side scatter and a lack of PI staining) represented a small fraction (1-2\%) of the detected events. Based on these data, the data from subsequent experiments were gated to include only viable cells, while excluding nonviable cells, cellular debris, and spores not associated with cells. Alternatively, the time dependent total uptake of spores was determined by plotting the geometric mean of the fluorescence intensity (MFI).

\section{Quantification of viable, intracellular B. anthracis}

Cells were incubated with dormant $B$. anthracis spores, as indicated above. For germinated $B$. anthracis spore infections, $B$. anthracis spore were germinated with 10 $\mathrm{mM} \mathrm{L}$-alanine and L-inosine in $1 \times \mathrm{PBS} \mathrm{pH} 7.2$ for 30 min and washed twice with $1 \times \mathrm{PBS} \mathrm{pH} 7.2$ to remove germinants and enumerated as described above. After 30 min, cells were washed three times with HBSS, and further incubated in the indicated medium with FBS $(10 \%)$ and gentamicin $(100 \mu \mathrm{g} / \mathrm{ml})$ to kill all external germinated spores. After $15 \mathrm{~min}$, the cells were washed three times with HBSS, and further incubated in the indicated appropriate medium supplemented with FBS $(10 \%)$. At the indicated times, the cells were lysed by incubating with sterile tissue culture grade water (Mediatech) for $5 \mathrm{~min}$ at $25^{\circ} \mathrm{C}$. Serial dilutions of the lysates were plated on LB agar plates and incubated overnight at $37^{\circ} \mathrm{C}$. CFU were enumerated by direct counting of visible colonies and correcting for the appropriate dilution.

\section{Statistics}

All data are representative of those from three or more independent experiments. The $Q$-test was performed to eliminate data that were statistical outliers [54]. Error bars represent standard deviations. $P$ values were calculated with Student's $t$ test using paired, one-tailed distribution. $P<0.05$ indicates statistical significance. Statistical analyses to calculate means, standard deviations, and Student's $t$ tests, were calculated using Microsoft Excel (version 11.0).

\section{Acknowledgements}

The authors would like to thank Dr. Barbara Pilas and Ben Montez from the R. J. Carver Biotechnology Center at the University of Illinois-Urbana/ Champaign (UIUC) for assistance with flow cytometry. This work was supported by an NIH-NIAID Award to the Western Regional Center for Excellence for Biodefense and Emerging Infectious Diseases Research U54Al057156 (SRB; P.I. D. Walker), a Chemical Biology Interface Training Grant from the National Institutes of Health (5 T32GM070421 to IMG), and by the Howard Hughes Medical Institute (to WAV).

\section{Author details}

${ }^{1}$ Department of Microbiology, University of Illinois, Urbana, IL, 61801, USA ${ }^{2}$ Department of Chemistry and Howard Hughes Medical Institute, University of Illinois, Urbana, IL, 61801, USA. Institute for Genomic Biology, University of Illinois, Urbana, IL, 61801, USA. ${ }^{4}$ Naval Medical Research Center, Biological Research Directorate, BDRD Annex, Rockville, MD, 20852, USA. ${ }^{5}$ Parkland College, Champaign, IL, 61821, USA.

\section{Authors' contributions}

IG assisted in experimental design, carried out the experiments, analyzed data, and drafted the manuscript. TB assisted in experimental design and data analysis, carried out the experiments, and assisted in drafting the manuscript. AP and BS conceived the study and performed preliminary experiments. SC carried out experiments. W helped to draft the manuscript. SB assisted in experimental design and drafted the manuscript. All authors read and approved the final manuscript.

Received: 29 November 2010 Accepted: 28 February 2011 Published: 28 February 2011

\section{References}

1. Dixon TC, Meselson M, Guillemin J, Hanna PC: Anthrax. N Engl J Med 1999, 341(11):815-826

2. Tournier JN, Quesnel-Hellmann A, Cleret A, Vidal DR: Contribution of toxins to the pathogenesis of inhalational anthrax. Cell Microbiol 2007, 9(3):555-565.

3. Frankel AE, Kuo SR, Dostal D, Watson L, Duesbery NS, Cheng CP, Cheng HJ, Leppla SH: Pathophysiology of anthrax. Front Biosci 2009, 14:4516-4524.

4. Cote CK, Rea KM, Norris SL, van Rooijen N, Welkos SL: The use of a model of in vivo macrophage depletion to study the role of macrophages during infection with Bacillus anthracis spores. Microb Pathog 2004, 37(4):169-175.

5. Cote CK, Van Rooijen N, Welkos SL: Roles of macrophages and neutrophils in the early host response to Bacillus anthracis spores in a mouse model of infection. Infect Immun 2006, 74(1):469-480.

6. Sanz P, Teel LD, Alem F, Carvalho HM, Darnell SC, O'Brien AD: Detection of Bacillus anthracis spore germination in vivo by bioluminescence imaging. Infect Immun 2008, 76(3):1036-1047.

7. Henderson DW, Peacock S, Belton FC: Observations on the prophylaxis of experimental pulmonary anthrax in the monkey. J Hyg (Lond) 1956, 54(1):28-36.

8. Cleret A, Quesnel-Hellmann A, Vallon-Eberhard A, Verrier B, Jung S, Vidal D, Mathieu J, Tournier JN: Lung dendritic cells rapidly mediate anthrax spore entry through the pulmonary route. J Immunol 2007, 178(12):7994-8001.

9. Shetron-Rama LM, Herring-Palmer AC, Huffnagle GB, Hanna P: Transport of Bacillus anthracis from the lungs to the draining lymph nodes is a rapid process facilitated by CD11c+ cells. Microb Pathog 2010, 49(1-2):38-46.

10. Russell BH, Vasan R, Keene DR, Koehler TM, Xu Y: Potential dissemination of Bacillus anthracis utilizing human lung epithelial cells. Cell Microbiol 2008, 10(4):945-957.

11. Russell BH, Vasan R, Keene DR, Xu Y: Bacillus anthracis internalization by human fibroblasts and epithelial cells. Cell Microbiol 2007, 9(5):1262-1274.

12. Russell BH, Liu Q, Jenkins SA, Tuvim MJ, Dickey BF, Xu Y: In vivo demonstration and quantification of intracellular Bacillus anthracis in lung epithelial cells. Infect Immun 2008, 76(9):3975-3983.

13. Dixon TC, Fadl AA, Koehler TM, Swanson JA, Hanna PC: Early Bacillus anthracis-macrophage interactions: intracellular survival survival and escape. Cell Microbiol 2000, 2(6):453-463.

14. Guidi-Rontani C, Mock M: Macrophage interactions. Curr Top Microbiol Immunol 2002, 271:115-141.

15. Guidi-Rontani C: The alveolar macrophage: the Trojan horse of Bacillus anthracis. Trends Microbiol 2002, 10(9):405-409.

16. Guidi-Rontani C, Levy M, Ohayon H, Mock M: Fate of germinated Bacillus anthracis spores in primary murine macrophages. Mol Microbiol 2001, 42(4):931-938.

17. Pickering AK, Osorio M, Lee GM, Grippe VK, Bray M, Merkel TJ: Cytokine response to infection with Bacillus anthracis spores. Infect Immun 2004, 72(11):6382-6389. 
18. Pickering AK, Merkel TJ: Macrophages release tumor necrosis factor alpha and interleukin-12 in response to intracellular Bacillus anthracis spores. Infect Immun 2004, 72(5):3069-3072.

19. Ruthel G, Ribot WJ, Bavari S, Hoover TA: Time-lapse confocal imaging of development of Bacillus anthracis in macrophages. J Infect Dis 2004, 189(7):1313-1316.

20. Welkos S, Friedlander A, Weeks S, Little S, Mendelson I: In-vitro characterisation of the phagocytosis and fate of anthrax spores in macrophages and the effects of anti-PA antibody. J Med Microbiol 2002, 51(10):821-831.

21. Kang TJ, Fenton MJ, Weiner MA, Hibbs S, Basu S, Baillie L, Cross AS: Murine macrophages kill the vegetative form of Bacillus anthracis. Infect Immun 2005, 73(11):7495-7501.

22. $\mathrm{Hu} \mathrm{H}, \mathrm{Sa} \mathrm{Q}$, Koehler TM, Aronson Al, Zhou D: Inactivation of Bacillus anthracis spores in murine primary macrophages. Cell Microbiol 2006, 8(10):1634-1642.

23. Guidi-Rontani C, Weber-Levy M, Labruyere E, Mock M: Germination of Bacillus anthracis spores within alveolar macrophages. Mol Microbiol 1999, 31(1):9-17.

24. Friedlander AM, Welkos SL, Pitt ML, Ezzell JW, Worsham PL, Rose KJ, Ivins BE, Lowe JR, Howe GB, Mikesell P, Lawrence WB: Postexposure prophylaxis against experimental inhalation anthrax. J Infect Dis 1993, 167(5):1239-1243.

25. Glomski IJ, Piris-Gimenez A, Huerre M, Mock M, Goossens PL: Primary involvement of pharynx and peyer's patch in inhalational and intestinal anthrax. PLOS Pathog 2007, 3(6):e76.

26. Drysdale M, Heninger S, Hutt J, Chen Y, Lyons CR, Koehler TM: Capsule synthesis by Bacillus anthracis is required for dissemination in murine inhalation anthrax. Embo J 2005, 24(1):221-227.

27. Zaucha GM, Pitt LM, Estep J, Ivins BE, Friedlander AM: The pathology of experimental anthrax in rabbits exposed by inhalation and subcutaneous inoculation. Arch Pathol Lab Med 1998, 122(11):982-992.

28. Oliva C, Turnbough CL Jr, Kearney JF: CD14-Mac-1 interactions in Bacillus anthracis spore internalization by macrophages. Proc Natl Acad Sci USA 2009, 106(33):13957-13962.

29. Oliva CR, Swiecki MK, Griguer CE, Lisanby MW, Bullard DC, Turnbough CL Jr, Kearney JF: The integrin Mac-1 (CR3) mediates internalization and directs Bacillus anthracis spores into professional phagocytes. Proc Natl Acad Sci USA 2008, 105(4):1261-1266.

30. Dozmorov M, Wu W, Chakrabarty K, Booth JL, Hurst RE, Coggeshall KM, Metcalf JP: Gene expression profiling of human alveolar macrophages infected by $B$. anthracis spores demonstrates TNF- $a$ and NF-kb are key components of the innate immune response to the pathogen. $B M C$ Infect Dis 2009, 9:152.

31. Xue Q, Jenkins SA, Gu C, Smeds $E_{1}$ Liu Q, Vasan R, Russell BH, Xu Y: Bacillus anthracis spore entry into epithelial cells is an actin-dependent process requiring c-Src and PI3K. PLOS One 2010, 5(7):e11665.

32. $\mathrm{Hu} \mathrm{H}$, Emerson J, Aronson Al: Factors involved in the germination and inactivation of Bacillus anthracis spores in murine primary macrophages. FEMS Microbiol Lett 2007, 272(2):245-250.

33. Bergman NH, Passalacqua KD, Gaspard R, Shetron-Rama LM, Quackenbush J, Hanna PC: Murine macrophage transcriptional responses to Bacillus anthracis infection and intoxication. Infect Immun 2005 73(2):1069-1080.

34. Sabet M, Cottam HB, Guiney DG: Modulation of cytokine production and enhancement of cell viability by TLR7 and TLR9 ligands during anthrax infection of macrophages. FEMS Immunol Med Microbiol 2006, 47(3):369-379.

35. Setlow P: Spore germination. Curr Opin Microbiol 2003, 6(6):550-556

36. Moir A, Corfe BM, Behravan J: Spore germination. Cell Mol Life Sci 2002, 59(3):403-409.

37. Moir A: How do spores germinate? J Appl Microbiol 2006, 101(3):526-530.

38. Levinson HS, Hyatt MT: Sequence of events during Bacillus megaterim spore germination. J Bacteriol 1966, 91(5):1811-1818.

39. Gut IM, Prouty AM, Ballard JD, van der Donk WA, Blanke SR: Inhibition of Bacillus anthracis spore outgrowth by nisin. Antimicrob Agents Chemother 2008, 52(12):4281-4288.

40. Ireland JA, Hanna PC: Macrophage-enhanced germination of Bacillus anthracis endospores requires gerS. Infect Immun 2002, 70(10):5870-5872

41. Fisher N, Hanna P: Characterization of Bacillus anthracis germinant receptors in vitro. J Bacteriol 2005, 187(23):8055-8062.
42. Barlass PJ, Houston CW, Clements MO, Moir A: Germination of Bacillus cereus spores in response to L-alanine and to inosine: the roles of gerL and gerQ operons. Microbiology 2002, 148(Pt 7):2089-2095.

43. Ireland JA, Hanna PC: Amino acid- and purine ribonucleoside-induced germination of Bacillus anthracis $\Delta$ Sterne endospores: gerS mediates responses to aromatic ring structures. J Bacteriol 2002, 184(5):1296-1303.

44. Paidhungat $M$, Setlow $P$ : Role of ger proteins in nutrient and nonnutrient triggering of spore germination in Bacillus subtilis. J Bacteriol 2000, 182(9):2513-2519.

45. Weiner MA, Read TD, Hanna PC: Identification and characterization of the gerH operon of Bacillus anthracis endospores: a differential role for purine nucleosides in germination. J Bacterio/ 2003, 185(4):1462-1464.

46. Stojkovic B, Torres EM, Prouty AM, Patel HK, Zhuang L, Koehler TM, Ballard JD, Blanke SR: High-throughput, single-cell analysis of macrophage interactions with fluorescently labeled Bacillus anthracis spores. Appl Environ Microbiol 2008, 74(16):5201-5210.

47. Banks DJ, Barnajian M, Maldonado-Arocho FJ, Sanchez AM, Bradley KA: Anthrax toxin receptor 2 mediates Bacillus anthracis killing of macrophages following spore challenge. Cell Microbiol 2005, 7(8):1173-1185.

48. Porasuphatana S, Cao GL, Tsai P, Tavakkoli F, Huwar T, Baillie L, Cross AS, Shapiro P, Rosen GM: Bacillus anthracis endospores regulate ornithine decarboxylase and inducible nitric oxide synthase through ERK1/2 and p38 mitogen-activated protein kinases. Curr Microbiol 2010, 61(6):567-573.

49. Shakir SM, Bryant KM, Larabee JL, Hamm EE, Lovchik J, Lyons CR, Ballard JD: Regulatory interactions of a virulence-associated serine/threonine phosphatase-kinase pair in Bacillus anthracis. J Bacteriol 2010, 192(2):400-409.

50. McKevitt MT, Bryant KM, Shakir SM, Larabee JL, Blanke SR, Lovchik J, Lyons CR, Ballard JD: Effects of endogenous D-alanine synthesis and autoinhibition of Bacillus anthracis germination on in vitro and in vivo infections. Infect Immun 2007, 75(12):5726-5734.

51. Bergman NH, Anderson EC, Swenson EE, Janes BK, Fisher N, Niemeyer MM, Miyoshi AD, Hanna PC: Transcriptional profiling of Bacillus anthracis during infection of host macrophages. Infect Immun 2007, 75(7):3434-3444

52. Coligan JE: Current Protocols in Immunology. Hoboken: John Wiley \& Sons; 1991.

53. Hed J, Hallden G, Johansson SG, Larsson P: The use of fluorescence quenching in flow cytofluorometry to measure the attachment and ingestion phases in phagocytosis in peripheral blood without prior cell separation. J Immunol Methods 1987, 101(1):119-125.

54. Dixon W: Analysis of extreme values. Ann Math Stat 1950, 21:488-506.

doi:10.1186/1471-2180-11-46

Cite this article as: Gut et al:: Bacillus anthracis spore interactions with mammalian cells: Relationship between germination state and the outcome of in vitro. BMC Microbiology 2011 11:46.

\section{Submit your next manuscript to BioMed Central and take full advantage of:}

- Convenient online submission

- Thorough peer review

- No space constraints or color figure charges

- Immediate publication on acceptance

- Inclusion in PubMed, CAS, Scopus and Google Scholar

- Research which is freely available for redistribution 